\title{
Functional Bosonization of Interacting Fermions in Arbitrary Dimensions
}

\author{
Peter Kopietz and Kurt Schőnhammer \\ Institut für Theoretische Physik der Universität Göttingen, \\ Bunsenstr.9, D-37073 Göttingen, Germany
}

(July 11, 1994)

\begin{abstract}
We bosonize the long-wavelength excitations of interacting fermions in arbitrary dimension by directly applying a suitable Hubbard-Stratonowich transformation to the Grassmannian generating functional of the fermionic correlation functions. With this technique we derive a surprisingly simple expression for the single-particle Greens-function, which is valid for arbitrary interaction strength and can describe Fermi- as well as Luttinger liquids. Our approach sheds further light on the relation between bosonization and the random-phase approximation, and enables us to study screening in a nonperturbative way.
\end{abstract}

PACS numbers: 05.30Fk, 05.30.Jp, 11.10.Ef, 71.27.+a

Several groups [1, 4 have recently constructed bosonization rules for interacting fermions in dimensions $d>1$. The strategy adopted in these works follows closely the usual bosonization of one-dimensional systems [5], and is based on the observation that suitably defined local density operators approximately obey bosonic commutation relations in the Hilbert space of states with wave-vectors close to the Fermi surface. An alternative method to bosonize interacting fermions without calculating commutators is based on functional integration [6.7]. In this letter we shall further develop this approach, and show that it is in many respects more powerful than the usual operator bosonization.

Consider a system of interacting fermions on a $d$ dimensional hypercube with volume $\mathcal{V}$. The hamiltonian is given by $\hat{H}=\hat{H}_{0}+\hat{H}_{\text {int }}$, with $\hat{H}_{0}=\sum_{\mathbf{k}} \epsilon_{\mathbf{k}} \hat{c}_{\mathbf{k}}^{\dagger} \hat{c}_{\mathbf{k}}$ and $\hat{H}_{\text {int }}=\frac{1}{2 \mathcal{V}} \sum_{\mathbf{q k k}^{\prime}} f_{\mathbf{q}}^{\mathbf{k k}^{\prime}} \hat{c}_{\mathbf{k}+\mathbf{q}}^{\dagger} \hat{c}_{\mathbf{k}^{\prime}-\mathbf{q}}^{\dagger} \hat{c}_{\mathbf{k}^{\prime}} \hat{c}_{\mathbf{k}}$, where $\hat{c}_{\mathbf{k}}$ annihilates an electron with wave-vector $\mathbf{k}$, and $\epsilon_{\mathbf{k}}$ is some arbitrary energy dispersion [8]. We assume that the degrees of freedom far away from the Fermi surface have been integrated out, so that $\mathbf{k}$ and $\mathbf{k}^{\prime}$ are restricted to a shell around the Fermi surface, with radial thickness small compared with the Fermi wave-vector $k_{F}$. Thus, $f_{\mathbf{q}}^{\mathbf{k k}^{\prime}}$ and $\epsilon_{\mathbf{k}}$ differ from the bare parameters by finite renormalizations. Following Refs. [1] [4], we partition the Fermi surface into disjoint patches of volume $\Lambda^{d-1}$. The precise shape of the patches is irrelevant as long as we are interested in fluctuations with wave-vectors $|\mathbf{q}| \ll \Lambda$, and the interaction $f_{\mathbf{q}}^{\mathbf{k k}^{\prime}}$ is dominated by momentum transfers $|\mathbf{q}| \ll k_{F}$. Throughout this work we shall assume that these restrictions are satisfied. Let us introduce a label $\alpha$ to enumerate the patches in some arbitrary ordering, and denote by $K^{\alpha}$ the set of k-points in patch $\alpha$. For each $\alpha$ we define local density operators
$\hat{R}_{\mathbf{q}}^{\alpha}=\sum_{\mathbf{k}} \Theta^{\alpha}(\mathbf{k}) \hat{c}_{\mathbf{k}}^{\dagger} \hat{c}_{\mathbf{k}+\mathbf{q}}$, where $\Theta^{\alpha}(\mathbf{k})=1$ if $\mathbf{k} \in K^{\alpha}$, and zero otherwise. Assuming that the variations of $f_{\mathbf{q}}^{\mathbf{k k}^{\prime}}$ are negligible if $\mathbf{k}$ and $\mathbf{k}^{\prime}$ are restricted to given patches, we may introduce the coarse-grained interaction function $f_{\mathbf{q}}^{\alpha \alpha^{\prime}}=\ll \Theta^{\alpha}(\mathbf{k}) \Theta^{\alpha^{\prime}}\left(\mathbf{k}^{\prime}\right) f_{\mathbf{q}}^{\mathbf{k k}^{\prime}} \gg$ where $\ll \ldots \gg$ denotes averaging with respect to $\mathbf{k}$ and $\mathbf{k}^{\prime}$.

To bosonize $\hat{H}$, we consider the imaginary-time correlation function

$$
\Pi_{q}^{\alpha \alpha^{\prime}}=\frac{1}{\mathcal{V}} \int_{0}^{\beta} d \tau e^{-i \omega_{m} \tau}<\hat{R}_{\mathbf{q}}^{\alpha}(\tau) \hat{R}_{-\mathbf{q}}^{\alpha^{\prime}}(0)>
$$

where $\beta=1 / T$ is the inverse temperature and $q=$ $\left[\mathbf{q}, i \omega_{m}\right]$ denotes wave-vector $\mathbf{q}$ and bosonic Matsubara frequency $\omega_{m}=2 \pi m T$. Below it will become evident that bosonization of $\hat{H}$ is equivalent to the calculation of a functional $S_{\text {eff }}\{\rho\}$ of a complex field $\rho_{q}^{\alpha}$, such that Eq.1] can be written as a bosonic functional integral

$$
\Pi_{q}^{\alpha \alpha^{\prime}}=\frac{\beta}{\mathcal{V}} \frac{\int \mathcal{D}\{\rho\} \rho_{q}^{\alpha} \rho_{-q}^{\alpha^{\prime}} e^{-S_{e f f}\{\rho\}}}{\int \mathcal{D}\{\rho\} e^{-S_{e f f}\{\rho\}}} .
$$

We now derive $S_{\text {eff }}\{\rho\}$ in arbitrary dimension. Starting point is the representation of $\Pi_{q}^{\alpha \alpha^{\prime}}$ as a functional integral over Grassmann fields $c_{k}$ [9]

$$
\Pi_{q}^{\alpha \alpha^{\prime}}=\frac{\beta}{\mathcal{V}} \frac{\int \mathcal{D}\{c\} R_{q}^{\alpha} R_{-q}^{\alpha^{\prime}} e^{-S_{1}\{c\}-S_{4}\{c\}}}{\int \mathcal{D}\{c\} e^{-S_{1}\{c\}-S_{4}\{c\}}},
$$

with $S_{1}\{c\}=\beta \sum_{k}\left[-i \tilde{\omega}_{n}+\xi_{\mathbf{k}}\right] c_{k}^{\dagger} c_{k}$, and $S_{4}\{c\}=$ $\frac{\beta}{2 \mathcal{V}} \sum_{q} \sum_{\alpha \alpha^{\prime}} R_{-q}^{\alpha} f_{\mathbf{q}}^{\alpha \alpha^{\prime}} R_{q}^{\alpha^{\prime}}$. Here $\tilde{\omega}_{n}=\pi(2 n+1) T$ are fermionic Matsubara frequencies, $\xi_{\mathbf{k}}=\epsilon_{\mathbf{k}}-\mu$ is the energy measured relative to the chemical potential $\mu$ of the interacting system, and $R_{q}^{\alpha}=\sum_{k} \Theta^{\alpha}(\mathbf{k}) c_{k}^{\dagger} c_{k+q}$. We now decouple $S_{4}\{c\}$ by means of a Hubbard-Stratonowich transformation that involves two auxiliary fields $\phi_{q}^{\alpha}$ and $\rho_{q}^{\alpha}$ [7]. Completing squares and using the invariance of the integration measure with respect to shifts [9], it is not difficult to show that

$$
e^{-S_{4}\{c\}}=\int \mathcal{D}\{\rho\} \mathcal{D}\{\phi\} e^{-S_{2}\{c, \phi\}-S_{3}\{\phi, \rho\}-S_{\text {int }}\{\rho\}},
$$

$$
\begin{aligned}
S_{2}\{c, \phi\} & =\sum_{k k^{\prime}} c_{k}^{\dagger} V_{k k^{\prime}} c_{k^{\prime}}, \quad V_{k k^{\prime}}=i \sum_{\alpha} \Theta^{\alpha}(\mathbf{k}) \phi_{k-k^{\prime}}^{\alpha} \\
S_{3}\{\phi, \rho\} & =-i \sum_{q} \sum_{\alpha} \phi_{-q}^{\alpha} \rho_{q}^{\alpha} \\
S_{\text {int }}\{\rho\} & =\frac{1}{2} \sum_{q} \sum_{\alpha \alpha^{\prime}} \rho_{-q}^{\alpha} \tilde{f}_{\mathbf{q}}^{\alpha \alpha^{\prime}} \rho_{q}^{\alpha^{\prime}}, \quad \tilde{f}_{\mathbf{q}}^{\alpha \alpha^{\prime}}=\frac{\beta}{\mathcal{V}} f_{\mathbf{q}}^{\alpha \alpha^{\prime}} .
\end{aligned}
$$


The field $\rho_{q}^{\alpha}$ is the bosonized local density field, while $\phi_{q}^{\alpha}$ is the field dual to $\rho_{q}^{\alpha}$. Because the composite fermionic field $R_{q}^{\alpha}$ couples to the dual field $\phi_{q}^{\alpha}$, the latter must be introduced at least at an intermediate step in order to be able to integrate the fermions out at the very beginning.

Because the effective fermionic action is quadratic, we may now perform the Grassmann integration, and obtain

$$
\begin{gathered}
\Pi_{q}^{\alpha \alpha^{\prime}}= \\
\frac{\beta}{\mathcal{V}} \frac{\int \mathcal{D}\{\rho\} \mathcal{D}\{\phi\} \rho_{q}^{\alpha} \rho_{-q}^{\alpha^{\prime}} e^{-S_{k i n}\{\phi\}-S_{3}\{\phi, \rho\}-S_{\text {int }}\{\rho\}}}{\int \mathcal{D}\{\rho\} \mathcal{D}\{\phi\} e^{-S_{k i n}\{\phi\}-S_{3}\{\phi, \rho\}-S_{\text {int }}\{\rho\}}},
\end{gathered}
$$

where $S_{k i n}\{\phi\}$ can be expanded as $S_{k i n}\{\phi\}=$ $\sum_{n=1}^{\infty} S_{k i n}^{(n)}\{\phi\}$, with $S_{k i n}^{(n)}\{\phi\}=\frac{1}{n \beta^{n}} \operatorname{Tr}\left[G_{0} V\right]^{n}$. The trace is over wave-vector and frequency space, and $G_{0}$ and $V$ are infinite matrices, with $\left[G_{0}\right]_{k k^{\prime}}=$ $\delta_{\mathbf{k}, \mathbf{k}^{\prime}} \delta_{n, n^{\prime}} G_{0}(k)$, and $G_{0}(k)=\left[i \tilde{\omega}_{n}-\xi_{\mathbf{k}}\right]^{-1}$. The matrixelements of $V$ are defined in Eq. 5 . The subscript on $S_{k i n}$ indicates that this quantity is closely related to the bosonized kinetic energy. Performing the trace in $S_{k i n}^{(n)}\{\phi\}$ yields

$$
\begin{aligned}
& S_{k i n}^{(n)}\{\phi\}=\frac{1}{n} \sum_{q_{1} \ldots q_{n}} \sum_{\alpha_{1} \ldots \alpha_{n}} \delta_{\mathbf{q}_{1}+\ldots+\mathbf{q}_{n}, 0} \delta_{m_{1}+\ldots+m_{n}, 0} \\
& \times U^{(n)}\left(q_{1} \alpha_{1} \ldots q_{n} \alpha_{n}\right) \phi_{q_{1}}^{\alpha_{1}} \cdots \phi_{q_{n}}^{\alpha_{n}} \\
& U^{(n)}\left(q_{1} \alpha_{1} \ldots q_{n} \alpha_{n}\right)=\left(\frac{i}{\beta}\right)^{n} \frac{1}{n !} \sum_{P} \sum_{k} G_{0}^{\alpha_{P_{1}}}(k) \\
& \times G_{0}^{\alpha_{P_{2}}}\left(k+q_{P_{1}}\right) \cdots G_{0}^{\alpha_{P_{n}}}\left(k+q_{P_{1}}+\ldots+q_{P_{n-1}}\right),
\end{aligned}
$$

where $G_{0}^{\alpha}(k)=\Theta^{\alpha}(\mathbf{k}) G_{0}(k)$. We have used the invariance of $S_{k i n}^{(n)}\{\phi\}$ under relabeling of the fields to symmetrize the vertices $U^{(n)}$ with respect to the interchange of any two labels. The sum $\sum_{P}$ is over the $n$ ! permutations of $n$ integers, and $P_{i}$ denotes the image of $i$ under the permutation. Note that the vertices $U^{(n)}$ are uniquely determined by $\xi_{\mathbf{k}}$. In $d>1$ or for one-dimensional models with non-linear energy dispersion, $U^{(n)}\left(q_{1} \alpha_{1} \ldots q_{n} \alpha_{n}\right)$ are rather complicated functions of all external momenta and frequencies. However, in the Tomonaga-Luttinger model (TLM) (i.e. for onedimensional fermions with linearized energy dispersion) all $U^{(n)}$ with $n \geq 3$ vanish identically. This is a direct consequence of the closed loop theorem, which is discussed and proved in Ref. 10]. Note that it is necessary to symmetrize the vertices in order to apply this theorem.

The first term in the expansion of $S_{k i n}\{\phi\}$ is $S_{\text {kin }}^{(1)}\{\phi\}=i \sum_{\alpha} N^{\alpha} \phi_{0}^{\alpha}$, where $N^{\alpha}=\sum_{\mathbf{k}} \Theta^{\alpha}(\mathbf{k}) f\left(\xi_{\mathbf{k}}\right)$ is the number of occupied states in patch $\alpha$. Here $f(\epsilon)=\left[e^{\beta \epsilon}+1\right]^{-1}$ is the Fermi function. For any finite $q$ the contribution from $S_{k i n}^{(1)}$ cancels in Eq.8. The second-order term is

$$
S_{k i n}^{(2)}\{\phi\}=\frac{1}{2} \sum_{q} \sum_{\alpha \alpha^{\prime}} \phi_{-q}^{\alpha} U^{(2)}\left(-q \alpha, q \alpha^{\prime}\right) \phi_{q}^{\alpha^{\prime}},
$$

where $U^{(2)}\left(-q \alpha, q \alpha^{\prime}\right)=\frac{\mathcal{V}}{\beta} \Pi_{0}^{\alpha \alpha^{\prime}}(q)$, with

$$
\begin{aligned}
& \Pi_{0}^{\alpha \alpha^{\prime}}(q)=\frac{1}{2 \mathcal{V}} \sum_{\mathbf{k}} {\left[\Theta^{\alpha}(\mathbf{k}) \Theta^{\alpha^{\prime}}(\mathbf{k}+\mathbf{q}) \frac{f\left(\xi_{\mathbf{k}}\right)-f\left(\xi_{\mathbf{k}+\mathbf{q}}\right)}{\xi_{\mathbf{k}+\mathbf{q}}-\xi_{\mathbf{k}}-i \omega_{m}}\right.} \\
&\left.+\left(\alpha \leftrightarrow \alpha^{\prime}, q \rightarrow-q\right)\right] .
\end{aligned}
$$

The higher order vertices describe non-local interactions between the fields. In the absence of nesting wave-vectors and Van Hove singularities, they are non-singular and have a finite limit if all external momenta and frequencies are set equal to zero [11]. In this limit $U^{(n)}$ is diagonal in all patch-labels, and is proportional to the $(n-2)^{n d}$ derivative of the density of states at the Fermi surface with respect to the chemical potential [11. Note that in the TLM the density of states is approximated by a constant, so that it is also from this point of view clear that $U^{(n)}\left(q_{i}=0\right)$ vanishes for $n \geq 3$. However, the closed loop theorem is stronger, and guarantees that the higherorder vertices vanish even at finite values of the $q_{i}$.

At this point we integrate over the field $\phi$. The effective action $S_{\text {eff }}\{\rho\}$ in Eq.2 is given by

$$
S_{\text {eff }}\{\rho\}=S_{\text {int }}\{\rho\}-\ln \left[\int \mathcal{D}\{\phi\} e^{-S_{k i n}\{\phi\}-S_{3}\{\phi, \rho\}}\right] .
$$

Performing the integration in Eq.13 perturbatively, we obtain an expansion $S_{\text {eff }}\{\rho\}=\sum_{n=1}^{\infty} S_{\text {eff }}^{(n)}\{\rho\}$, where $S_{\text {eff }}^{(n)}\{\rho\}$ is of the same form as Eq 9 , but with the $\phi$-fields replaced by the $\rho$-fields, and the vertices $U^{(n)}\left(q_{1} \alpha_{1} \ldots q_{n} \alpha_{n}\right)$ replaced by a new set of vertices $\Gamma^{(n)}\left(q_{1} \alpha_{1} \ldots q_{n} \alpha_{n}\right)$. In general $\Gamma^{(n)}$ contains contributions from all $U^{(m)}$ with $m \geq n$. We now truncate the expansion of $S_{k i n}\{\phi\}$ at the second order. In the TLM this approximation is exact, while in $d>1$ it is expected to be accurate at high densities and for models where the higher-order derivatives of the density of states are small. The integration in Eq.13 is then Gaussian, and we obtain

$$
S_{e f f}^{(2)}\{\rho\}=\frac{1}{2} \sum_{q} \sum_{\alpha \alpha^{\prime}} \rho_{-q}^{\alpha}\left[\tilde{f}_{\mathbf{q}}^{\alpha \alpha^{\prime}}+\Gamma^{(2)}\left(-q \alpha, q \alpha^{\prime}\right)\right] \rho_{q}^{\alpha^{\prime}}
$$

where $\Gamma^{(2)}$ is the matrix inverse to $U^{(2)}$ in the space spanned by the patch indices. Since we are interested in long-wavelength fluctuations, we may approximate $\Gamma^{(2)}$ by its leading term for small $|\mathbf{q}|$. At high densities $U^{(2)}\left(-q \alpha, q \alpha^{\prime}\right) \propto \delta^{\alpha \alpha^{\prime}}$, because the sum in Eq.12 is dominated by momenta $\mathbf{k}$ of the order of $k_{F}$, so that we may set $\Theta^{\alpha}(\mathbf{k}) \Theta^{\alpha^{\prime}}(\mathbf{k}+\mathbf{q}) \approx \delta^{\alpha \alpha^{\prime}} \Theta^{\alpha}(\mathbf{k})$ in Eq.12. In this approximation we obtain

$$
\Gamma^{(2)}\left(-q \alpha, q \alpha^{\prime}\right) \approx \delta^{\alpha \alpha^{\prime}} \frac{\beta}{\mathcal{V} \nu^{\alpha}} \frac{\xi_{\mathbf{q}}^{\alpha}-i \omega_{m}}{\xi_{\mathbf{q}}^{\alpha}},
$$

where $\nu^{\alpha}=\mathcal{V}^{-1} \partial N^{\alpha} / \partial \mu$ is the local density of states in patch $\alpha$, and $\xi_{\mathbf{q}}^{\alpha}=\mathbf{v}^{\alpha} \cdot \mathbf{q}$, with $\mathbf{v}^{\alpha}=\left.\nabla_{\mathbf{k}} \epsilon_{\mathbf{k}}\right|_{\mathbf{k} \in K^{\alpha}}$. Inserting Eq.15 into Eq.14, we see that the term proportional 
to $i \omega_{m}$ defines the dynamics of the $\rho$-field. We now recall that in the functional integral for canonically quantized bosons the coefficient of the term proportional to $-i \omega_{m}$ should be precisely $\beta$. Thus, to write our effective action in terms of a canonical boson field $b_{q}^{\alpha}$, we should rescale the $\rho$-field accordingly. This is achieved by substituting $\rho_{q}^{\alpha}=\left(\mathcal{V}^{\alpha}\left|\xi_{\mathbf{q}}^{\alpha}\right|\right)^{\frac{1}{2}}\left[\Theta\left(\xi_{\mathbf{q}}^{\alpha}\right) b_{q}^{\alpha}+\Theta\left(-\xi_{\mathbf{q}}^{\alpha}\right) b_{-q}^{\dagger \alpha}\right]$ in Eq.14. Our final result for the bosonized action $S^{(2)}\{b\} \equiv S_{\text {eff }}^{(2)}\{\rho(b)\}$ is

$$
\begin{gathered}
S^{(2)}\{b\}=\beta \sum_{q} \sum_{\alpha} \Theta\left(\xi_{\mathbf{q}}^{\alpha}\right)\left(-i \omega_{m}\right) b_{q}^{\alpha \dagger} b_{q}^{\alpha} \\
+\beta\left[H_{k i n}\{b\}+H_{\text {int }}\{b\}\right], \\
H_{k i n}\{b\}=\sum_{q} \sum_{\alpha} \Theta\left(\xi_{\mathbf{q}}^{\alpha}\right) \xi_{\mathbf{q}}^{\alpha} b_{q}^{\alpha \dagger} b_{q}^{\alpha}, \\
H_{\text {int }}\{b\}=\frac{1}{2} \sum_{q} \sum_{\alpha \alpha^{\prime}} \Theta\left(\xi_{\mathbf{q}}^{\alpha}\right) \sqrt{\left|\xi_{\mathbf{q}}^{\alpha} \| \xi_{\mathbf{q}}^{\alpha^{\prime}}\right|} \\
\times\left[\Theta\left(\xi_{\mathbf{q}}^{\alpha^{\prime}}\right)\left(\bar{f}_{\mathbf{q}}^{\alpha \alpha^{\prime}} b_{q}^{\alpha \dagger} b_{q}^{\alpha^{\prime}}+\bar{f}_{\mathbf{q}}^{\alpha^{\prime} \alpha} b_{q}^{\alpha^{\prime} \dagger} b_{q}^{\alpha}\right)\right. \\
\left.+\Theta\left(-\xi_{\mathbf{q}}^{\alpha^{\prime}}\right)\left(\bar{f}_{\mathbf{q}}^{\alpha \alpha^{\prime}} b_{q}^{\alpha \dagger} b_{-q}^{\alpha^{\prime} \dagger}+\bar{f}_{\mathbf{q}}^{\alpha^{\prime} \alpha} b_{-q}^{\alpha^{\prime}} b_{q}^{\alpha}\right)\right],
\end{gathered}
$$

where $\bar{f}_{\mathbf{q}}^{\alpha \alpha^{\prime}}=\sqrt{\nu^{\alpha} \nu^{\alpha^{\prime}}} f_{\mathbf{q}}^{\alpha \alpha^{\prime}}$ are dimensionless couplings. The functional integral for the $b$-field is now formally identical with a standard bosonic functional integral. The corresponding second quantized bosonic hamiltonian is therefore $\hat{H}^{b}=\hat{H}_{k i n}^{b}+\hat{H}_{i n t}^{b}$, where $\hat{H}_{k i n}^{b}$ and $\hat{H}_{i n t}^{b}$ are simply obtained by replacing the bosonic fields $b_{q}^{\alpha}$ in Eqs.17, 18 by operators $\hat{b}_{\mathbf{q}}^{\alpha}$ satisfying $\left[\hat{b}_{\mathbf{q}}^{\alpha}, \hat{b}_{\mathbf{q}^{\prime}}^{\alpha^{\prime} \dagger}\right]=\delta^{\alpha \alpha^{\prime}} \delta_{\mathbf{q}, \mathbf{q}^{\prime}}$. The resulting $\hat{H}^{b}$ agrees with the bosonized Hamiltonian derived in Refs. 3., 便 by means of an operator approach. From our derivation it is obvious that bosonization is a non-perturbative but approximate method in $d>1$. The non-interacting boson-approximation is only accurate if the higher order terms $S_{k i n}^{(n)}, n \geq 3$, are irrelevant.

$\hat{H}^{b}$ and $S_{\text {eff }}^{(2)}\{\rho\}$ (Eq.14) contain the same physical information. To calculate $\Pi_{q}^{\alpha \alpha^{\prime}}$, it is convenient to parametrize the functional integration in terms of the original density field $\rho_{q}^{\alpha}$. Inserting Eq.14 into Eq.22 we obtain $\Pi_{q}^{\alpha \alpha^{\prime}}=\left[\left[\Pi_{0}{ }^{-1}(q)+f_{\mathbf{q}}\right]^{-1}\right]^{\alpha \alpha^{\prime}}$ where $\Pi_{0}$ and $f$ should be understood as matrices in the patch labels. This is nothing but the random-phase approximation (RPA), which is known to be exact in the TLM [12]. The physical density-density correlation function is $\sum_{\alpha \alpha^{\prime}} \Pi_{q}^{\alpha \alpha^{\prime}}$. In $d>1$ the summation over the patches gives rise to Landau-damping.

Let us now focus on the single-particle Greens-function $G^{\alpha}(\mathbf{r}, \tau)$ associated with patch $\alpha[13]$. It is well-known [11] that the Hubbard-Stratonowich transformation reduces the calculation of $G^{\alpha}(\mathbf{r}, \tau)$ to the problem of calculating the Greens-function $\mathcal{G}^{\alpha}\left(\mathbf{r}, \mathbf{r}^{\prime}, \tau, \tau^{\prime} ;\{\phi\}\right)$ of an effective non-interacting system in a dynamical randomfield $\phi$. The crucial observation is that after linearization of the energy dispersion $\mathcal{G}^{\alpha}$ can be calculated exactly for a given realization of $\phi$, because the differential equation for $\mathcal{G}^{\alpha}$ is first order and can be easily solved [6]. The physical Greens-function is then obtained by averaging $\mathcal{G}^{\alpha}$ over the distribution of the field $\phi$. Within the non-interacting boson approximation, this average involves a trivial Gaussian integration. The final result for the real-space imaginary-time Greens-function is $G^{\alpha}(\mathbf{r}, \tau)=G_{0}^{\alpha}(\mathbf{r}, \tau) e^{Q^{\alpha}(\mathbf{r}, \tau)}$, with

$$
\begin{aligned}
& Q^{\alpha}(\mathbf{r}, \tau)=R^{\alpha}-S^{\alpha}(\mathbf{r}, \tau), R^{\alpha}=\lim _{\mathbf{r}, \tau \rightarrow 0} S^{\alpha}(\mathbf{r}, \tau), \\
& S^{\alpha}(\mathbf{r}, \tau)=\frac{1}{\beta \mathcal{V}} \sum_{q} \frac{\left[f_{q}^{R P A}\right]^{\alpha \alpha} \cos \left(\mathbf{q} \cdot \mathbf{r}-\omega_{m} \tau\right)}{\left(i \omega_{m}-\xi_{\mathbf{q}}^{\alpha}\right)^{2}} .
\end{aligned}
$$

Here $f_{q}^{R P A}=\left[f_{\mathbf{q}}^{-1}+\Pi_{0}(q)\right]^{-1}$ is again a matrix in the patch indices. Eqs.19 and 20 can be used to determine by direct calculation of the Greens-function if the system is a Fermi- or Luttinger liquid, or perhaps belongs even to a different category. In Refs. [4, 14 the Greensfunction is also found to be of the form $G^{\alpha}=G_{0}^{\alpha} e^{Q^{\alpha}}$. Note, however, that Ref. [⿴囗十 and Ref. [14] give different expressions for $Q^{\alpha}$. Our $Q^{\alpha}$ resembles more the result derived by Castellani et al. via Ward identities, although it is not precisely the same, because their $Q^{\alpha}$ depends explicitly on an ultraviolet cutoff. In our approach all cutoff-dependence is contained in the local density of states $\nu^{\alpha}$. Below we show that for a quite general class of interactions Eq.20 depends only on the global density of states $\nu=\sum_{\alpha} \nu^{\alpha}$, and is therefore manifestly cutoff-independent. We now discuss Eqs.19 and 20 for $\mathcal{V}, \beta \rightarrow \infty$ in some physically interesting cases. A detailed derivation will be given in a longer publication.

(a) Luttinger liquid in $d=1$. In one dimension Eqs.19 and 20 correctly reproduce the known results for the Greens-function of the TLM. Note that $R^{\alpha}$ and $S^{\alpha}(\mathbf{r}, \tau)$ are both logarithmically divergent if $f_{\mathbf{q}}^{\alpha \alpha^{\prime}}$ goes to a finite limit for $\mathbf{q} \rightarrow 0$, but $Q^{\alpha}(\mathbf{r}, \tau)$ is finite. It seems that the possibility of expressing the interaction dependence of $Q^{\alpha}(\mathbf{r}, \tau)$ exclusively in terms of the RPA-interaction has not been noticed in the literature on the TLM.

(b) Quasi-particle residue. If the integral defining $R^{\alpha}$ exists, the system is by the usual definition a Fermi liquid. The quasi-particle residue $Z^{\alpha}$ for wave-vectors $\mathbf{k} \in K^{\alpha}$ is then $Z^{\alpha}=e^{R^{\alpha}}$. From Eq.20 it is not difficult to show that $R^{\alpha}$ is real and negative, so that $0<Z^{\alpha} \leq 1$.

(c) Singular interactions. For interactions of the form $f_{\mathbf{q}}^{\alpha \alpha^{\prime}}=g^{2}|\mathbf{q}|^{-\eta} e^{-|\mathbf{q}| / q_{c}}, \eta \geq 0$, we find by simple power counting that $R^{\alpha}$ exists only for $\eta<2(d-1)$, in agreement with Ref. [15]. If in addition $\frac{d-1}{2}<\eta$, and if the screening wave-vector $\kappa=\left(\nu g^{2}\right)^{1 / \eta}$ is small compared with $k_{F}$, then the ultraviolet cutoff $q_{c}$ is not necessary and may be set to infinity, because $\kappa$ acts as a natural short-distance cutoff for all wave-vector integrals. It is important to stress that we are not a priori assuming that the bare interaction is screened. A straight-forward calculation gives in this case for the quasi-particle residue 


$$
Z^{\alpha}=\exp \left[-\frac{r(d, \eta)}{2(d-1)-\eta}\left(\kappa / k_{F}\right)^{d-1}\right],
$$

where the positive numerical constant $r(d, \eta)$ can be explicitly written down as a one-dimensional integral, and remains finite at $\eta=2(d-1)$.

(d) Coulomb interaction in $d>1$. This is a special case of (c): $g^{2}=s_{d} e^{2}, \eta=d-1$, and $q_{c}=\infty$. Here $s_{d}$ is a numerical constant $\left(s_{2}=2 \pi, s_{3}=4 \pi\right) . \quad \kappa=$ $\left(\nu s_{d} e^{2}\right)^{\frac{1}{d-1}}$ can now be identified with the usual ThomasFermi screening wave-vector. Note that $\kappa \ll k_{F}$ in the high-density limit we are considering here, and that $\eta=$ $d-1$ lies for all $d$ in the regime $\frac{d-1}{2}<\eta<2(d-1)$ where $\kappa$ acts as an intrinsic ultraviolet cutoff. We conclude that at high densities the Coulomb gas is a Fermi liquid in all physical dimensions $d>1$. The quasi-particle residue can be obtained by setting $\eta=d-1$ in Eq.21. If we analytically continue to non-integer dimensions 14], we see that $Z^{\alpha} \ll 1$ for $d-1 \ll\left(\kappa / k_{F}\right)^{d-1}$. It can be shown that $r(d, d-1) \sim \frac{1}{2}+O(d-1)$ for small $d-1$, so that $Z^{\alpha} \propto\left(k_{F} / \kappa\right)^{1 / 2} \exp \left[-\frac{1}{2(d-1)}\right]$ for $d \rightarrow 1$.

(e) Quasi-particle damping. If $\left|R^{\alpha}\right|<\infty$ and the integrand in Eq.20 is a sufficiently smooth function of $q$, the Fourier integral theorem implies $\left|S^{\alpha}(\mathbf{r}, \tau)\right|<\infty$ for all $\mathbf{r}$ and $\tau$, and $\lim _{\mathbf{r}, \tau \rightarrow \infty} S^{\alpha}(\mathbf{r}, \tau)=0$. The precise way in which $S^{\alpha}(\mathbf{r}, \tau)$ vanishes determines the quasiparticle damping. For a conventional Fermi liquid with damping $\gamma_{\mathbf{q}}^{\alpha} \propto\left(\xi_{\mathbf{q}}^{\alpha}\right)^{2}$ the leading term vanishes for large $u \equiv \max \left\{|\mathbf{r}|,\left|\mathbf{v}^{\alpha} \tau\right|\right\}$ as $u^{-1}$. For interactions of the form discussed in (c) we find that $S^{\alpha}(\mathbf{r}, \tau) \sim c_{1} u^{-(d-1-\eta / 2)}+$ $c_{2} u^{-1}$ as $u \rightarrow \infty$, where $c_{1}$ and $c_{2}$ are constants. For $d<2+\frac{\eta}{2}$ the first term decays slower than the second one, and gives rise to anomalously large corrections. In particular, the Coulomb interaction satisfies this condition for $d<3$, which includes the physically accessible case $d=2$. The vanishing of $S^{\alpha}(\mathbf{r}, \tau)$ for large $u$ implies that the effective mass is not renormalized. Thus, the definition of $\epsilon_{\mathbf{k}}$ in our original hamiltonian $\hat{H}_{0}$ should take the effective mass renormalization into account.

(f) Relevance of transverse hopping. Experiments probing the Luttinger liquid regime are performed on anisotropic quasi one-dimensional conductors, consisting of weakly coupled chains embedded in a threedimensional lattice. If the nature of the threedimensional interaction $f_{\mathbf{q}}^{\alpha \alpha^{\prime}}$ is such that for vanishing interchain hopping $t_{\perp}$ the system is a Luttinger liquid, then for any finite $t_{\perp}$ the quasi-particle residue is finite, so that strictly speaking these systems are not Luttinger liquids. If $t_{\perp}$ is small compared with the characteristic intra-chain hopping energy $t_{\|}$, then the quasi-particle residue is proportional to $\left(t_{\perp} / t_{\|}\right)^{\gamma}$, where the non-universal exponent $\gamma>0$ can be identified with the anomalous dimension of the Luttinger liquid that would exist for $t_{\perp}=0$ [16].

Finally, we would like to point out that our approach opens the way for a systematic calculation of corrections to the non-interacting boson approximation, which exist even in $d=1$ if the energy dispersion is not linearized [5].
Treating the terms $S_{k i n}^{(n)}$ for $n \geq 3$ perturbatively within one-loop approximation, we find that for interactions of the form $f_{\mathbf{q}}^{\alpha \alpha^{\prime}}=f_{0} e^{-|\mathbf{q}| / q_{c}}, q_{c} \ll k_{F}$, the non-Gaussian corrections to the bosonized hamiltonian are negligible if the dimensionless parameter $A_{0}=q_{c}^{d} \frac{f_{0}}{1+\nu f_{0}}\left|\frac{\partial \nu}{\partial \mu}\right|$ is small compared with unity. Note that $A_{0}$ vanishes if the energy dispersion is linearized, because then the density of states is approximated by a constant independent of $\mu$.

We would like to thank G. E. Castilla and V. Meden for discussions and comments on the manuscript.

[1] A. Luther, Phys. Rev. B 19, 320 (1979).

[2] F. D. M. Haldane, Helv. Phys. Acta. 65, 152 (1992).

[3] A. Houghton et al., Phys. Rev. B 50, 1351 (1994); ibid. 48, 7790 (1993); J. Phys. 6, 4909 (1994).

[4] A. H. Castro Neto and E. Fradkin, Phys. Rev. Lett. 72, 1393 (1994); Phys. Rev. B 49, 10877 (1994); and preprint (1994).

[5] F. D. M. Haldane, J. Phys. C 14, 2585 (1981).

[6] H. C. Fogedby, J. Phys. C 9, 3757 (1976).

[7] D. K. K. Lee and Y. Chen, J. Phys. A 21, 4155 (1988).

[8] For simplicity we have suppressed the spin label. The spin is easily taken into account by defining $\mathbf{k}$ and $\mathbf{k}^{\prime}$ to be collective labels for wave-vector and spin.

[9] V. N. Popov, Functional Integrals and Collective Excitations, (Cambridge University Press, Cambridge, 1987).

[10] T. Bohr, Nordita preprint 81/4, Lectures on the Luttinger Model, 1981 (unpublished). As realized in these notes, the $\mathbf{q}=0$ part of the interaction requires a special treatment.

[11] J. A. Hertz and M. A. Klenin, Phys. Rev. B 10, 1084 (1974).

[12] I. E. Dzyaloshinskii and A. I. Larkin, Zh. Eksp. Teor. Fiz. 65, 411 (1973) [Sov. Phys. JETP 38, 202 (1974)].

[13] $G^{\alpha}(\mathbf{r}, \tau)=\frac{1}{\beta \mathcal{V}} \sum_{k} e^{i\left(\mathbf{k} \cdot \mathbf{r}-\tilde{\omega}_{n} \tau\right)} \Theta^{\alpha}(\mathbf{k}) G\left(\mathbf{k}, i \tilde{\omega}_{n}\right)$, where $G\left(\mathbf{k}, i \tilde{\omega}_{n}\right)$ is the usual Matsubara Greens-function. Note that the non-interacting function $G_{0}^{\alpha}(\mathbf{r}, \tau)$ is proportional to $\delta^{(d-1)}\left(\mathbf{r}_{\perp}^{\alpha}\right)$, where $\mathbf{r}_{\perp}^{\alpha}$ is the $d-1$ dimensional projection of $\mathbf{r}$ perpendicular to $\mathbf{v}^{\alpha}$. Therefore we may set $\mathbf{r}=x \mathbf{v}^{\alpha} /\left|\mathbf{v}^{\alpha}\right|$ in Eq.20.

[14] C. Castellani et al., Phys. Rev. Lett. 72, 316 (1994); and preprint (1994).

[15] P.-A. Bares and X.-G. Wen, Phys. Rev. B 48, 8636 (1993).

[16] P. Kopietz, V. Meden, and K. Schőnhammer, preprint (August 1994). 